\title{
Social Navigation Support for Information Seeking: If You Build It, Will They Come?
}

\author{
Rosta Farzan and Peter Brusilovsky \\ Intelligent Systems Program \\ University of Pittsburgh \\ Pittsburgh, PA, 15260 \\ rosta@cs.pitt.edu, peterb@mail.sis.pitt.edu
}

\begin{abstract}
Navigating through the ever-changing information space is becoming increasingly difficult. Social navigation support is a technique for guiding users to interesting and relevant information by leveraging the browsing behavior of past users. Effect of social navigation support on users' information seeking behavior has been studied mostly from conceptual basis or under natural experiments. In the current work, we have designed and conducted a controlled experiment to investigate the effect of social navigation support through a multifaceted method. This paper reports on the design of the study and the result of log data, subjective evaluation, and eye movement data analysis.
\end{abstract}

\section{Introduction}

Social Navigation emerged into a popular research area at the crossroads of two active research fields - personalized information access and social Web. Social navigation assists users browsing through Web resources by applying "community wisdom" distilled from the actions of earlier users. This navigation support most frequently comes in the form of visual cues indicating, for example, which of the available links were picked by the majority of similar users [22], or which pages were being explored by other users at the moment [14].

Despite the popularity of social navigation ideas [11], very few studies of social navigation systems can be found in the research literature. The majority of research done in the field falls into two categories of (1) Conceptual structure which focus on theoretical discussion of social navigation phenomena and design aspects with little or no focus on evaluation; and (2) Natural experiments that rely solely on observations of the effect of social navigation on the users' navigation behavior in the system under study rather than manipulating variables in controlled experiments. As a result, while there is a popular belief that social navigation support (SNS) is powerful and helpful, we know very little about the value of various social navigation approaches. Moreover, we are not sure whether the users of social navigation systems follow social navigation cues or these cues are simply ignored. In our past research [7], we attempted to present some evidence that social visual cues are noticed and used. Our results, however, were based on user log data collected during a long-term classroom study and can be 
considered rather as the first step in exploring the impact of social navigation on user behavior. The click-stream collected in the user logs caused us to rely on secondary evidence about user attention to visual cues. A user click on an annotated link may be caused by the usefulness of the link, not the attached social cue. At the same time, the lack of a click does not really mean that the visual cue was not noticed - the link could be simply less relevant to the user in a specific context.

The work presented in this paper attempted to explore on a deeper level the impact of social navigation cues on users' information seeking behavior. We have designed a controlled experiment to assess the following questions: Do the users notice social navigation cues? Do the provided cues affect and change their link selection? Do the visual cues become more useful under time pressure when the user has little time to make a proper navigation decision? The experiment focused on a factual information seeking task designed for a lab study. We observed users' information seeking behavior with and without SNS and time constraint. We extended observational and log data by using eyetracking. Eye tracking data provides information about users' areas of interest and attention and helps to closely examine the effect of social navigation cues on users' information seeking behavior.

The rest of the paper explains the design of the study and presents part of the results of the study. We conclude with a discussion of the results and their implications for the design of similar systems and plans for the future work.

\section{Background}

User navigation can be called social when it is driven by the actions from one or more advice providers [5]. In its classic form, social navigation attempts to visualize the aggregated or individual actions of a user community to help future users navigate through complex information spaces such as the Web. Social navigation in information spaces as well as the term social navigation were introduced by Dourish and Chalmers as "moving towards cluster of people" or "selecting objects because others have examined them" [5]. However, the idea of social navigation is frequently traced back to the pioneer Edit Wear and Read Wear systems [10]. Hill and Hollan introduced the idea of physical wear in the domain of document processing as "computational wear". Computational wear is the visualization of the history of authors' and readers' interactions with a document. The visualization of the history enables the new users to quickly locate the most viewed or edited parts of the document.

The systems Juggler [4] and Footprints [22] are classical examples which used social navigation to help users navigating in two kinds of information spaces a Web site and a text-based virtual environment. Both systems attempted to visualize traces to guide future users. Wexelblat and Mayes [22] introduced the idea of interaction history for digital information which is taken from extensive use of history traces in the physical world. Footprints provides contextualized navigation through usage of several interface features such as maps, path views, 
annotations, and sign posts. Juggler is an educational tool which combines a textbased virtual environment (known as MOO) and a Web browser. Juggler highlights major navigation paths through different textual bulletin boards (rooms) and adds the computational wear to each bulletin boards by showing the number of times it was accessed. Juggler also supports a direct form of social navigation by encouraging users to directly recommend useful resources (such as URLs) to each other. Another example of a system with several forms of social navigation is KALAS [21], a food recipe system. It provides a history-enriched environment by visualizing the aggregated trail of users through the environment. The trail includes the comments left by the users as well as information about the number of users who have downloaded a recipe. KALAS supports direct social navigation by displaying currently logged on users in each section of the system and allowing real-time chat among the users. Implementation of social navigation goes beyond these classical examples. Growth of social information access applications on the Web over the last decade suggests social navigation as a response to problem of disorientation on the Web [20], [15], [6].

While the idea of social navigation has widely been implemented, evaluation of the effect of social navigation support is a less explored area. Evaluation methodologies used to evaluate information seeking tools can be employed to achieve deeper insight into the effectiveness and strength of social navigation support. Recently researchers in the field of information retrieval have been attracted to utilizing eye tracking for better understanding of users' search behavior and to model users and their interests beyond log analysis and queries they type in. Accurate viewing is only possible in 1-2 degrees of visual angle. As a result, gaze direction is a reliable indicator of the focus of attention.

Eye movement data are typically divided into fixations and saccades. Fixations are relative pauses of eye movements over an informative region of interest while saccades are the rapid eye movements between the fixations [19]. The main methodology employed in interface evaluation using eye-tracking is through dividing the interface into predefined areas of interest [8] and collecting users' eye movement on those areas. Number of fixations, location of fixations, fixation duration, and cumulative fixation time are some of the most commonly used measures in evaluation of computer interfaces using eye-tracking [18], [12]. Joachims et al [13] extended the work on assessing the reliability of implicit feedback by detailed analysis of users' decision making process through the use of eye tracking. They analyzed users' fixations on the search results page to understand how to associate users' decision process with their clickthrough actions and how to generate feedback from clicks. In a similar study, Cutrell and Guan [3] used eye tracking to investigate how users attend to different parts of web search results and whether users' search strategies are different for navigational versus informational tasks. Specifically, they were interested on assessing the effect of snippet length on how people use Web search. Chi et al [2] studied the eye-gaze behavior of subjects to understand how highlighting keywords and sentences containing highly relevant conceptual keywords (ScentHighlights) affected subjects' reading behavior. They were interested in assessing whether highlighting is successful in 
directing users' attention while skimming the text. They analyzed users' initial fixations and eye behavior, and percentage of fixations on highlighted areas.

\section{Study of Social Navigation Support}

In the current work, we explored the role of several factors on the added value of SNS. We designed an experiment to investigate the circumstances under which SNS can help users in an information exploration task. In the experiment, we controlled the presence of SNS and time to complete the task. We were specifically interested in investigating the effect of time pressure on the usage of SNS.

\subsection{Task}

The study investigated the effect of SNS on a factual information seeking task which is known as the most common type of information seeking task on the Web [17]. The participants were asked to respond to several questions by finding facts in a very large collection of relevant and irrelevant news articles from multiple sources. In our past work, we developed a set of search tasks (topics) for this collection and collected passage-level ground truth for each topic [9]. Each topic contains an overarching task theme and up to 10 different (but related) factual questions. Over the course of the study the participants worked with four topics of our collection solving four different search tasks. For each search task they were given a one-page task description providing a brief background to the task scenario and a list of questions to answer (a subset of 10 questions). To minimize the impact of topics, we tried to pick the topics with a similar difficulty level. We used data from a related prior study to judge the difficulty level of the topics [1]. We used criteria such as number of relevant documents in the corpus, average number of relevant documents returned by users, and number of questions to match the difficulty level of the topics.

\section{$3.2 \quad$ Interface}

For our study, we developed an information exploration system with social navigation. Figure 1 shows the main interface of the system. It is similar to other search engines in which the user enters a query and the results are returned sorted by the relevance to the query. In addition to this traditional interface, the experimental version of the system offers two kinds of social navigation support. First, the search results are annotated with social navigation cues. The cues are based on two types of user activities: reading and highlighting. The human icon represents the amount of reading activity for the associated document and the annotation icon represents the amount of highlighting done in the document. The level of the filling color represents the density of the activity with a higher level of filling representing the higher number of activities. Mousing over the icons shows the details about the number of visits or number of highlights. 


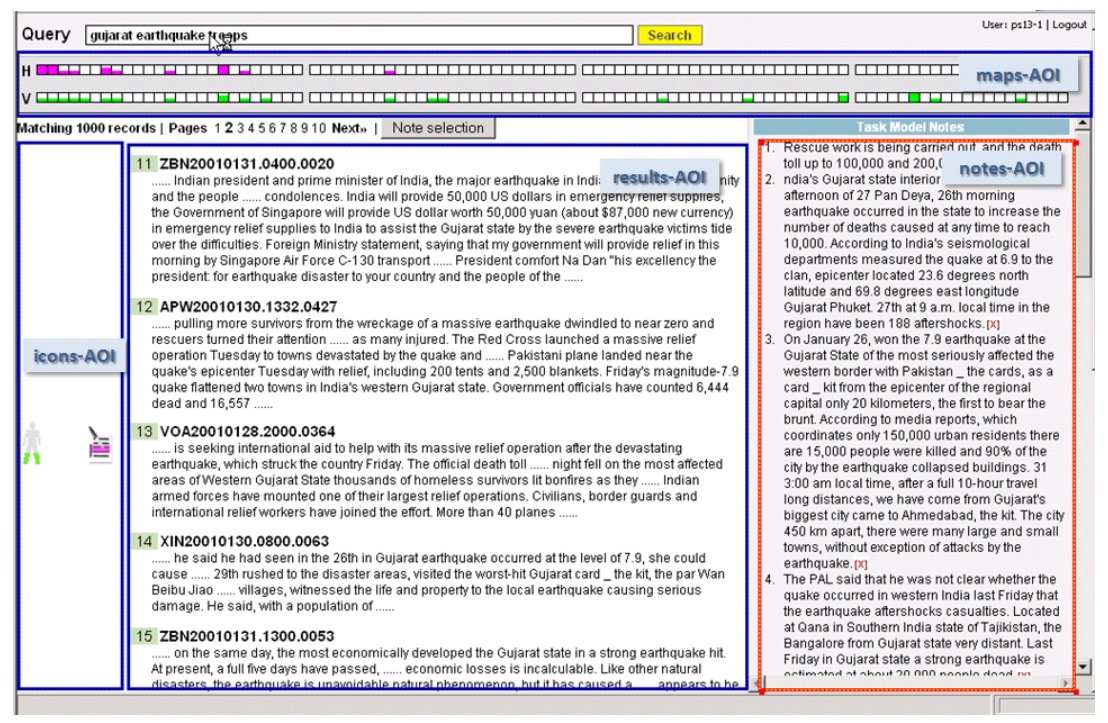

Fig. 1. Search Interface (with eye tracking areas of interest)

Second kind of social navigation support is offered by "social maps". Social maps are two tables at the top of the page, representing highlighting and visiting activities for the current 100 documents. Each cell in the table is associated with the document with the same rank as the cell number; i.e. first one is associated with the first document in the list. Users can directly access the document by clicking on the map cell. The filling level of the cell represents the magnitude of the activity. If the cell is empty, it means the associated document has not been visited or highlighted by anyone. The social maps provide information beyond 10 documents returned on each page of search results. They were designed to help users have broader picture of the results in an easy way.

A panel on the right side of the interface shows the list of notes (passages) collected by the current user. To collect notes, users can highlight and save a passage either directly from a snippet shown for each returned document in the list of search results or from the full text of the article, which the user can open by clicking on the document title. The passages saved from this document by past users will be highlighted providing another level of SNS. Figure 2 shows an example of the full text of the article. At this view, SNS is offered by showing the part of the text highlighted by past users. By default, other users' highlighted passages are shown (in pink). Users can choose to ignore that information and view their own highlighted passages (in yellow).

Social Navigation Support The system provided SNS through augmenting search results with icons, social maps, and previously highlighted parts of the text. In real life, social navigation cues are generated from the activity of all past users. However, this is not good for a controlled study since every new user may see more cues than past users. To avoid this, in our study we used 


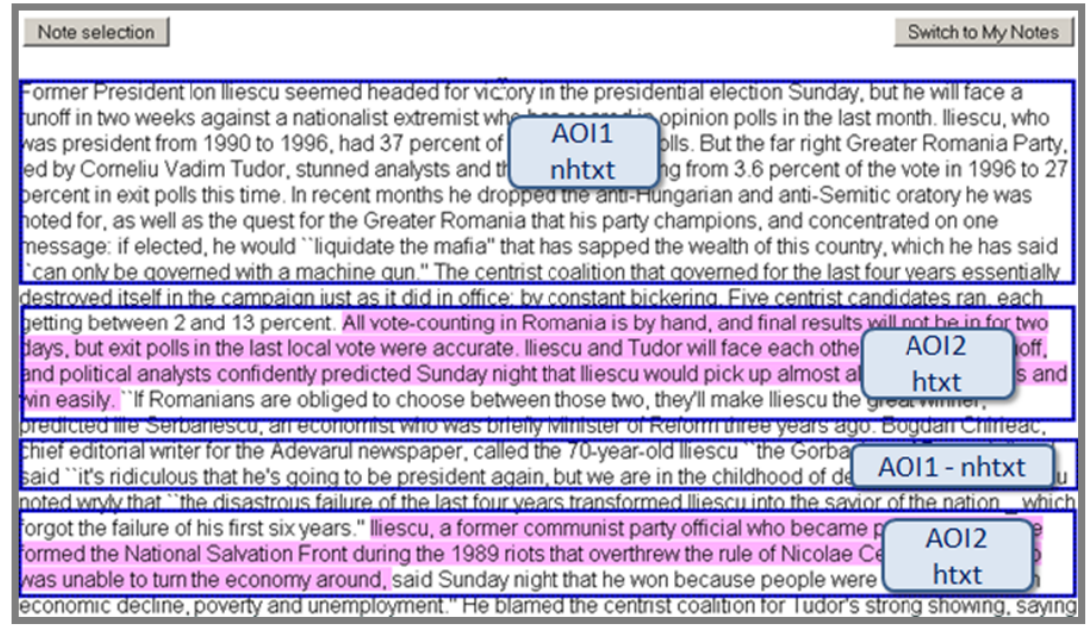

Fig. 2. Full text of the article (with eye tracking areas of interest)

"frozen" social cues generated from the activity of users from a prior study [1]. For each topic, the data includes the activities of three distinct users. To make the task more realistic, we divided the questions for each topic into two sets with the criteria of decreasing within-cluster similarity and increasing betweencluster similarity. This simulated a collaborative task which is divided among the members of a group. We calculated the similarity of two questions based on the shared number of documents which included the response to each of the questions. To cluster the questions, we calculated the similarity of questions for every possible distinct combination of five questions in a set. We selected the sets with the highest between-cluster similarity and lowest within-cluster similarity. This means that SNS will guide the users to the right articles but the highlighted parts of the articles are not necessarily responses to questions in their task. SNS was not updated with the interaction history of the participants throughout the study. This ensured that all users have the same opportunity of getting support from social navigation cues.

\subsection{Study Design}

The study has a two-by-two design as shown in Table 1. It follows a complete random design in which the order of conditions and topics are selected randomly. Under no time constraint condition the participants had 15 minutes and under time constraint they had seven minutes. NO-SNS condition had no social navigation cues. The interface looked the same as Figure 1 but with no social navigation icons and no social maps. Also, when they looked at the full article, there was no option to view passages highlighted by prior users.

The procedure of the study was at follows: first a brief description about the experiment was provided. Next an eye tracking calibration was done to ensure 
Title Suppressed Due to Excessive Length

Table 1. Study design - conditions

\begin{tabular}{|l|c|c|c|}
\hline \multicolumn{2}{|c|}{} & \multicolumn{2}{|c|}{ SNS } \\
\hline \multirow{2}{*}{ Time Constraint } & Yes (tc group) & Topic 1 & No (no-SNS group) \\
& No (no-tc group) & Topic 3 & Topic 2 \\
\end{tabular}

reasonable precision in tracking eye movements followed by a demographic and skill questionnaire which included questions about the participants' age, gender, major, and educational level. The questionnaire also included five questions measuring interpersonal trust adopted from a questionnaire by Mooradian et al [16]. The questions are shown in Table 2. The response to all questions had five choices ranging from "Strongly agree" to "Strongly disagree". Different parts of the interface were explained thoroughly to all subjects and they all went through a training session to become familiar with the interface before starting the main task. Each session included the main task and a subjective evaluation of the participants' satisfaction with the system.

Table 2. Interpersonal Trust Questionnaire

\begin{tabular}{ll}
\hline \# & Question \\
\hline 1 & I tend to be cynical and skeptical of others' intentions \\
2 & I believe that most people will take advantage of you if you let them \\
3 & If I got into difficulties at work I know my colleagues would try and help me out \\
4 & I can trust the people I work with to lend me a hand if I needed it \\
5 & Most of my peers can be relied upon to do as they say they will do \\
\hline
\end{tabular}

\section{Evaluation}

We recruited 15 participants from students at the University of Pittsburgh from several different disciplines including engineering, information science, life sciences, and humanities. Participants were paid for their participation in the study. To limit the variability of linguistic abilities, we recruited native English speakers. Nine out of 15 participants were female. Their age ranged from 20 to 35 with the average age equal to $24(\sigma=5)$.

\subsection{Eye Tracking Data Analysis}

We defined two main stimuli, "search result" and "text" as shown in Figure 1 and Figure 2. Search result stimulus includes four areas of interest (AOIs) (1) Icons, (2) Maps, (3) Results, and (4) Notes. We were interested to measure the percentage of fixations and gaze time on AOI1 and AOI2 to assess the influence of SNS on users' search behavior. The text stimulus includes two AOIs: (1) not highlighted text (AOI1-nhtxt) and (2)highlighted text (AOI2-htxt). Here, we were interested in measuring the percentage of fixations and gaze time on highlighted parts of the text to assess the influence of presenting prior users' highlights on current users' reading and highlighting behavior. Due to time constraints we analyzed eye movements data of five participants out of 15 who went through the experiment. 


\subsection{Statistical Analysis}

Since the study deals with correlated data we performed hypothesis testing with models designed for correlated data. We fitted three types of models with respect to the distribution of the response variable and goodness of fit: (1) Linear, (2) Negative Binomial, and (3) Gamma.

\subsection{Hypotheses and Results}

The main goal of the evaluation was to assess the effect of SNS on participants' search behavior specifically with and without time constraint. The following are the main research questions we have tried to address in the analysis of the study:

- Q1 - Were participants more likely to click on documents augmented with social navigation cues? Were they more likely to follow SNS under time pressure?

- Q2 - Are eye movement data going to be different in terms of following social navigation cues under time constraint?

- Q3 - How much of the participants' highlighting and reading behavior was influenced by already highlighted text?

Question 1 To answer the first question, we calculated the average percentage of clicks on documents with and without SNS icons for each subject and we conducted repeated measure analysis to check whether there is a difference in terms of number of clicks. The result shows a significant effect of social navigation cues: documents augmented with cues were accessed significantly more (Wald $\chi^{2}=24.16, \mathrm{df}=1, \mathrm{p}$-value $\left.<.001\right)$. Additionally, the result shows a significant effect of $S N S$ condition and significant interaction of $S N S$ and social navigation cues which means augmented documents were accessed more under SNS condition (SNS: Wald $\chi^{2}=4.70, \mathrm{df}=1, \mathrm{p}$-value $=.030, \mathrm{SNS} \times$ augmented: Wald $\chi^{2} 6.86$, $\mathrm{df}=1$, $\mathrm{p}$-value $=.009)$. We emphasize that under the no-SNS condition, the participants were not aware of which documents could have been augmented. This result is important to show that augmented documents are not just the important documents that would have been accessed even if not augmented. There is marginal significance of time pressure which suggests participants were slightly more likely to click on augmented documents under the time constraint condition (Wald $\chi^{2}=3.14, \mathrm{df}=1$, $\mathrm{p}$-value $=.076$ ).

Question 2 We hypothesized that users under time pressure will be in more need of navigation support and will make more use of SNS. Number of fixations and total gaze time on the social navigation icons and social navigation maps can be an indication of how much they have utilized those navigation supports. We calculated the percentage of fixation count and gaze time on those AOIs while users were looking at search results. The average percentage is shown in Figure 3. The result shows a similar number of fixations and amount of gaze 


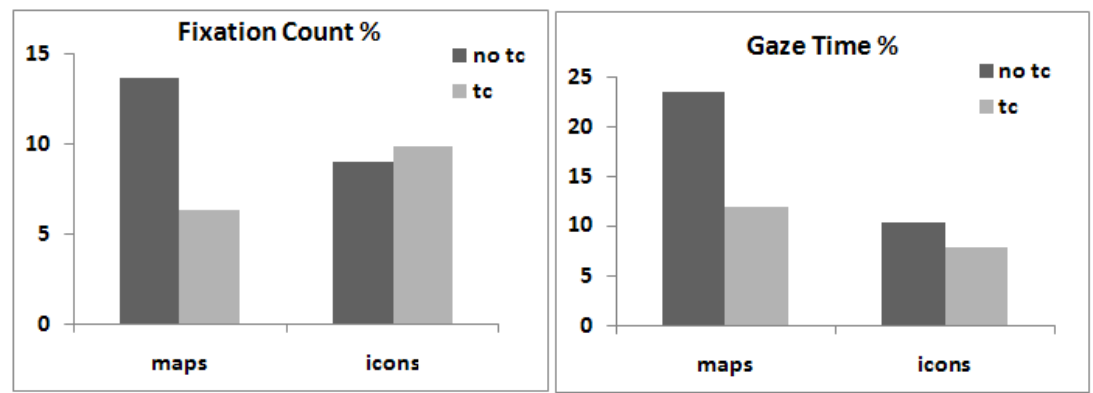

Fig. 3. Average percentage of fixations and gaze time on social navigation AOIs

time over social navigation icons and a higher number of fixations and amount of gaze time on social navigation maps under the no time constraint condition.

This is an interesting result which does not match our expectation. This can be due to the fact that users under the time constraint condition had time to check very few articles and they might have mainly relied on the rank of search results. They still relied on social navigation cues integrated with the ranked list and checked the icons. However, they did not have enough time to explore anything (like social navigation maps) beyond highly-ranked results. In fact, our data shows that under the time constraint condition participants selected articles from significantly higher (numerically lower) ranking (Average rank: no-tc $=4.2$, $\mathrm{tc}=1.8)$.

Question 3 As we mentioned before, the highlighted text was not entirely related to questions the current participants were working on; instead there were responses to similar questions. We were interested in assessing how much the reading and highlighting behavior of participants were affected by those highlights and whether there is going to be a large overlap between their collected passages and highlighted passages. To answer this question, we calculated the percentage of overlapping notes for each participant as number of characters overlapping the highlighted area divided by total length of notes selected by that participant. On average, there was $9.3 \%$ overlap under time constraint condition $(\sigma=.04)$ and $5.7 \%$ overlap under no time constraint condition $(\sigma=.09)$. There is no significant effect of the condition on the average overlap. The result suggests that while the users were slightly influenced by highlights, they did not just select notes from highlighted areas. Our eye-tracking data supports the same result. The average percentage of fixation count is significantly higher on not highlighted text as compared to highlighted text(htxt-AOI: $\mu=18.92$, nhtxt-AOI: $\mu=80.51$, Wald $\chi^{2}=37.014, \mathrm{df}=1, \mathrm{p}$-value $<.0001$ ). The result shows that users did not only focus on highlighted text and spent considerable amount of time reading not highlighted text. It suggests that SNS helped the participants to get to the relevant documents, but within a document they relied on their own judgment. 


\subsection{Subjective Evaluation}

We conducted a survey after each session to evaluate users' subjective opinions about the system. The first five questions were the same after all sessions: they asked about general usability of the system and whether the users had enough time to perform the task.

The result shows that, under all four conditions, it was quite easy to find relevant documents and passages. However, under the NO-SNS and time constraint condition, the participants were less happy with the output of the system for answering the questions (There is a marginal significance interaction of SNS and time constraint - wald $\chi^{2} 2=3.03, \mathrm{df}=1, \mathrm{p}$-value=.08). While our eye movements and click-stream data shows that the participants did not necessarily utilize social navigation cues more under time pressure, this result suggests that presence of social navigation cues was somehow reassuring for them.

Additionally, the survey after the $S N S$ conditions collected the users' opinion about SNS. The questions are shown in Table 3. The possible response to all questions ranged from 1 (not at all) to 5 (extremely).

Table 3. Subjective evaluation - SNS related questions

\begin{tabular}{l}
\hline \# Question \\
\hline 1 Did you find it useful to know what documents were selected by other users? \\
2 Did you find it useful to know what documents were highlighted by other users? \\
3 Did you find it useful to view passages highlighted by other users? \\
4 Did you find it useful to know the number of times each document was visited? \\
5 Did you find it useful to know the number of times each document was highlighted? \\
6 The tables on top of the page were designed to facilitate navigating to documents highlighted \\
or visited by other users. Did you find it useful?
\end{tabular}

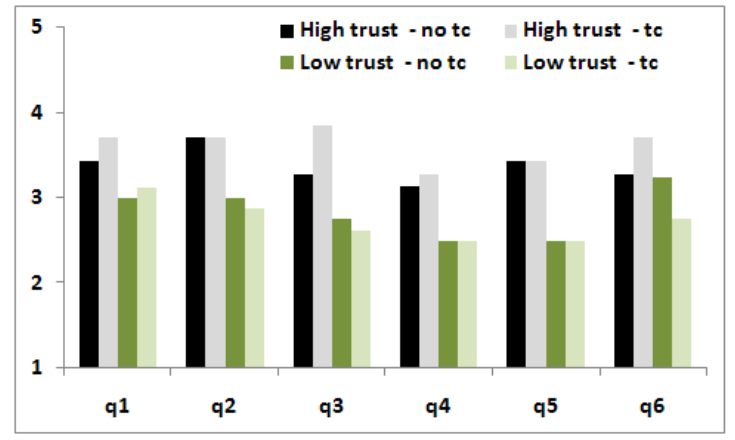

Fig. 4. Average responses to SNS related questions in the subjective evaluation 
As mentioned earlier, as part of our demographic questionnaire, we measured participants' interpersonal trust level. We divided the participants into high trust and low trust levels based on their responses to those questions. Eight participants were in the low trust level and seven in the high trust group. We were interested to determine any effect of interpersonal trust on their judgment of the usefulness of SNS. Figure 4 shows the average responses to the SNS-related questions of the questionnaire. The response is divided into four groups depending on the trust level and time constraint. The result suggests that participants with higher interpersonal trust levels are more satisfied with SNS, particularly under time constraint.

\section{Discussion and Future Work}

In the current work, we presented a multifaceted study of SNS in a controlled experiment designed for factual information seeking tasks. The result of the study confirms that social navigation cues affect users' search behavior and users pay attention to social navigation cues and follow those cues for finding information. However, contrary to our expectation, time constraint did not increase the applicability of SNS and traditional navigational support such as search rank proved to be more reliable for users. The result also shows that personal characteristics such as interpersonal trust affects the perception of usefulness of SNS. This should to be taken into account for user modeling applications. Moreover, our result suggests that the snowball effect often associated with social navigation can be avoided if the systems offer users sufficient information to make their own informed judgement. In our study, the participants did not select part of the text just because it was highlighted by prior users and even though the highlights were slightly relevant to their task.

An element missing in the current study is assessing the effect of SNS on the quality of the performed task. For our future work, we will look into evaluating the effect of SNS on the relevancy of collected information to the task. Moreover, we will extend our eye movement data analysis to a larger number of users to to assess our current observations with larger amount of data.

\section{References}

1. J. Ahn, P. Brusilovsky, D. He, J. Grady, and Q. Li. Personalized web exploration with task models. In Proceedings of the 17th international conference on World Wide Web, 2008.

2. E. H. Chi, M. Gumbrecht, and L. Hong. Visual foraging of highlighted text: An eyetracking study. In Proceeding of Human-Computer Interaction (HCII'O7), volume 4552, pages 589-598. Springer-Verlag, 2007.

3. E. Cutrell and Z. Guan. What are you looking for? an eye-tracking study of information usage in web search. In Proceedings of the SIGCHI conference on Human factors in computing systems, 2007.

4. A. Dieberger. Supporting social navigation on the world wide web. International Journal of Human-Computer Interaction, 46:805-825, 1997. 
5. P. Dourish and M. Chalmers. Running out of space: Models of information navigation. In Proceedings of HCI'94, 1994.

6. J. Dron. Termites in the schoolhouse: Stigmergy and transactional distance in an e-learning environment. In L. Cantoni and C. McLoughlin, editors, Proceedings of World Conference on Educational Multimedia, Hypermedia and Telecommunications, pages 263-269, Chesapeake, VA, 2004. AACE.

7. R. Farzan and P. Brusilovsky. Annotated: A social navigation and annotation service for web-based educational resources. New Review in Hypermedia and Multimedia, 14(1):3-32, 2008.

8. J. H. Goldberg and X. P. Kotval. Computer interface evaluation using eyemovement: methods and constructs - its psychological foundation and relevance to display design. International Journal of Industrial Ergomics, 1999.

9. B. P. A. J.-W. G. J. F. R. P. Y. Y. Y. He, D. and M. Rogati. An evaluation of adaptive filtering in the context of realistic task-based information exploration. Information Processing and Management, 44(2):511-533, 2008.

10. W. C. Hill, J. D. Hollan, D. Wroblewski, and T. Mccandless. Edit wear and read wear. In P. Bauersfeld, J. Bennett, and G. Lynch, editors, Proceedings of the SIGCHI conference on Human factors in computing systems, pages 3-9, 1992.

11. K. Höök, D. Benyon, and A. J. Munro, editors. Designing Information Spaces: The Social Navigation Approach. Berlin: Springer, 2003.

12. R. J. K. Jacob and K. S. Karn. Eye tracking in human computer interaction and usability research: ready to deliver the promises (section commentary). The minds eye: cognitive and applied aspects of eye movement research, pages 573-605, 2003.

13. T. Joachims, L. Granka, and B. Pan. Accurately interpreting clickthrough data as implicit feedback. In SIGIR'05, 2005.

14. J. Kurhila, M. Miettinen, P. Nokelainen, and H. Tirri. Educo - a collaborative learning environment based on social navigation. In Springer, editor, Proceedings of 2nd International Conference on Adaptive Hypermedia and Adaptive Web-Based Systems, pages 242-252, 2002.

15. D. R. Millen, J. Feinberg, and B. Kerr. Dogear: Social bookmarkin in the enterprise. In Proceedings of CHI, 2006.

16. T. Mooradian, B. Renzl, and K. Matzler. Who trusts? personality, trust and knowledge sharing. Management Learning, 37(4):523-540, 2006.

17. J. Morrison, P. Pirolli, and S. K. Card. A taxonomic analysis of what world wide web activities significantly impact people's decisions and actions. In Proceedings of CHI 2001, 2001.

18. A. Poole and L. J. Ball. Encyclopedia of Human Computer Interaction, chapter Eye Tracking in Human-Computer Interaction and Usability Research: Current Status and Future Prospects. Information Science Reference, 2006.

19. K. Rayner. Eye movements in reading and information processing: 20 years of research. Psychological Bulletin, 124:372-422, 1998.

20. B. Smyth, E. Balfe, J. Freyne, P. Briggs, M. Coyle, and O. Boydell. Exploiting query repetition and regularity in an adaptive community-based web search engine. User Modeling and User-Adaptated Interaction, 14(5):383-423, 2004.

21. M. Svensson, K. Hk, J. Laaksolahti, and A. Waern. Social navigation of food recipes. In Proceedings of CHI, Seattle, April 2001. ACM.

22. A. Wexelblat and P. Maes. Footprints: History-rich tools for information foraging. In Proceeding of ACM Conference on Human-Computer Interaction (CHI'99), pages 270-277. ACM Press, 1999. 